\title{
Effects of cigarette smoke and hypoxia on pulmonary circulation in the guinea pig
}

\author{
E. Ferrer*, V.I. Peinado*,\#, J. Castañeda ${ }^{\#, \uparrow, ~ J . ~ P r i e t o-L l o r e t ", ~}{ }^{\#, \text { E. Olea }}{ }^{\#, \text {, }}$ \\ M.C. González-Martín\#,ף, M.V. Vega-Agapito „, M. Díez*, D. Domínguez-Fandos*,

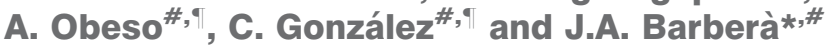

ABSTRACT: Cigarette smoke (CS) and chronic hypoxia $(\mathrm{CH})$ can produce pulmonary hypertension. Similarities and differences between both exposures and their interaction have not been explored. The aim of the present study was to investigate the effects of $\mathrm{CS}$ and $\mathrm{CH}$, as single factors or in combination, on the pulmonary circulation in the guinea pig.

51 guinea pigs were exposed to CS for 12 weeks and 32 were sham-exposed. $50 \%$ of the animals in each group were additionally exposed to $\mathrm{CH}$ for the final 2 weeks. We measured pulmonary artery pressure $(\mathrm{Ppa})$, and the weight ratio between the right ventricle $(\mathrm{RV})$ and left ventricle plus the septum. Pulmonary artery contractility in response to noradrenaline (NA), endothelium-dependent vasodilatation and distensibility were evaluated in organ bath chambers. The number of small intrapulmonary vessels showing immunoreactivity to smooth muscle (SM) $\alpha$-actin and double elastic laminas was assessed microscopically.

$\mathrm{CS}$ and $\mathrm{CH}$ induced similar increases of $P$ pa and RV hypertrophy ( $<<0.05$ for both), effects that were further enhanced when both factors were combined. $\mathrm{CH}$ increased the contractility to NA $(p<0.01)$ and reduced the distensibility $(p<0.05)$ of pulmonary arteries. Animals exposed to CS showed an increased number of small vessels with positive immunoreactivity to SM $\alpha$-actin $(p<0.01)$ and those exposed to $\mathrm{CH}$ a greater proportion of vessels with double elastic laminas $(p<0.05)$.

We conclude that $\mathrm{CH}$ amplifies the detrimental effects of $\mathrm{CS}$ on the pulmonary circulation by altering the mechanical properties of pulmonary arteries and enhancing the remodelling of pulmonary arterioles.

KEYWORDS: Chronic obstructive pulmonary disease, cigarette smoke, hypoxia, pulmonary hypertension, vascular remodelling

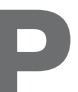

ulmonary hypertension is a common and serious complication of chronic obstructive pulmonary disease (COPD). It is considered to result from the effects of chronic hypoxaemia on pulmonary vessels. Indeed, acute and chronic exposure to hypoxia results in smooth muscle cell (SMC) and adventitial fibroblast proliferation [1]. Nevertheless, structural changes in the pulmonary arteries of COPD patients differ from those observed in subjects exposed to a hypoxic environment. Whereas subjects living at high altitudes show medial hypertrophy, COPD patients show prominent changes in the intima [2]. Intimal hypertrophy is present in nonhypoxaemic COPD patients and in smokers with normal lung function, suggesting that vascular changes may be triggered by cigarette smoke (CS) before hypoxaemia develops.
Cigarette smoking is associated with endothelial dysfunction [3], increased expression of growth factors [4] and inflammatory cell infiltrate in pulmonary arteries [5]. These factors may induce SMC proliferation and increase pulmonary vascular resistance. The molecular mechanisms by which CS induces vascular changes remain unknown, but they might be related to oxidative damage [6].

The effects of hypoxia on human lungs are difficult to characterise because it is usually related to the presence of primary lung diseases that may be associated with vascular remodelling by additional mechanisms (e.g. inflammation). Nevertheless, there is evidence that in humans, chronic hypoxia $(\mathrm{CH})$ per se may induce endothelial dysfunction [7], increased expression of growth factors [8] and inflammation [9].
AFFILIATIONS

${ }^{*}$ Dept of Pulmonary Medicine, Hospital Clínic-IDIBAPS, Universitat de Barcelona, Barcelona,

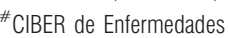
Respiratorias, and "Dept of Biochemistry and Molecular Biology and Physiology, and IBGM, Universidad de Valladolid, Consejo Superior de Investigaciones Científicas, Valladolid, Spain.

CORRESPONDENCE

J.A. Barberà

Dept of Pulmonary Medicine

Hospital Clínic

Villarroel 170

08036 Barcelona

Spain

E-mail: jbarbera@clinic.ub.es

Received:

July 062010

Accepted after revision: Jan 092011

First published online: Feb 102011 
Some of the observations in pulmonary vessels of COPD patients have been replicated in a model in which guinea pigs are chronically exposed either to CS or hypoxia. Exposure to CS induces muscularisation of pre-capillary vessels and increases pulmonary artery pressure $(P$ pa $)[10,11]$, which runs in parallel to endothelial dysfunction $[12,13]$ and develops before pulmonary emphysema is apparent. $\mathrm{CH}$, however, induces pulmonary hypertension, right ventricle (RV) hypertrophy, vascular remodelling [14] and increased plasma levels of endothelin (ET)-1 in the guinea pig [15].

We have hypothesised that in COPD, changes in pulmonary vessels are initiated at early disease stages as a result of a direct effect of CS products on the pulmonary endothelium. Subsequently, with the progression of the disease, hypoxaemia may develop, producing further vascular damage potentially resulting in pulmonary hypertension. The combined effects of $\mathrm{CS}$ and $\mathrm{CH}$ on the pulmonary circulation have not yet been assessed in experimental models.

Accordingly, the present study aimed to evaluate the effects of $\mathrm{CS}$ exposure and $\mathrm{CH}$, alone and as combined stimuli, on pulmonary haemodynamics, RV structure, pulmonary vascular reactivity and vessel remodelling in the guinea pig.

\section{METHODS}

Additional details on methods used are provided in the online supplementary material.

\section{Animals and management}

83 male Hartley guinea pigs weighing $350 \mathrm{~g}$ were divided into four groups: one group was exposed to CS for 12 weeks $(n=33)$; a second group was kept in a normal atmosphere for 10 weeks and subsequently exposed to an inspiratory oxygen fraction $\left(\mathrm{FI}, \mathrm{O}_{2}\right)$ of 0.12 for 2 weeks in a hypoxic chamber $(\mathrm{CH}$ group) ( $n=16)$; a third group was exposed to CS for 12 weeks and to $\mathrm{CH}$ for the final 2 weeks (CSCH group) $(n=18)$; and a control group was sham-exposed to CS and kept in a normal atmosphere $(n=16)$.

\section{CS exposure}

Animals were exposed to the smoke of four research cigarettes (2R4F; Kentucky University Research, Lexington, KY, USA) per day, 5 days week $^{-1}$ for 12 weeks using a nose-only system $[13,16]$.

\section{$\mathrm{CH}$ exposure}

Animals were placed in hypoxic glass chambers continuously fluxed with gas mixture (12\% oxygen in nitrogen; oxygen tension $\sim 85 \mathrm{mmHg}$, which is equivalent to an altitude of $\sim 4,300 \mathrm{~m}$ ).

\section{Pulmonary haemodynamics}

In half of the animals in each group, a catheter was placed in the pulmonary artery through the RV. The right carotid artery was canulated in order to simultaneously measure systemic arterial pressure (SAP). Measurements were performed in normoxic and hypoxic conditions (4 $\mathrm{min}$ at an $\mathrm{FI}_{1} \mathrm{O}_{2}$ of 0.10 ). The recovery profile was evaluated by pumping in air with an $\mathrm{F}, \mathrm{O}_{2}$ of 0.21 .

\section{Vessel distensibility and endothelial function}

Half of the animals in each group underwent assessment of vessel distensibility and reactivity. Vessel distensibility was evaluated in rings of pulmonary artery and aorta (length $3 \mathrm{~mm}$ ) as distensibility $=\Delta D / \Delta P \cdot D$, where $\Delta D$ is the difference of diameter before and after $1 \mathrm{~mm}$ of stretching, $\Delta P$ is the difference in pressure at the same points and $D$ is the final diameter of the ring $[17,18]$. Endothelial function was assessed as the change in wall tension in response to cumulative doses of adenosine diphosphate (ADP), as previously described [13].

\section{Macro- and microscopic morphologic studies}

$\mathrm{RV}$ hypertrophy was measured as the ratio between the RV weight and the weight of the left ventricle plus the septum (Fulton index). Wall thickness of the aorta and main pulmonary artery were measured [13]. In order to further assess the characteristics of the extracellular matrix of the vessel wall, the area occupied by mucopolysaccharides, collagen and elastin was evaluated in adjacent sections of the main pulmonary artery stained with Alcian blue, Masson trichrome and orcein, respectively, and expressed as a percentage of total vessel area.

The number of intrapulmonary vessels with a diameter $<50 \mu \mathrm{m}$ showing positive immunostaining for smooth muscle (SM) $\alpha$-actin was counted and expressed as percentage of the total number of small vessels. These vessels were further classified as nonmuscularised, partially muscularised or fully muscularised, according to the proportion of the vessel wall positive for SM $\alpha$-actin [19]. Additionally, the number of intrapulmonary vessels $<50 \mu \mathrm{m}$ in diameter with double elastic laminas, assessed in orcein-stained sections, were counted and expressed as a percentage of the total number of small intrapulmonary vessels.

\section{Assessment of cell proliferation}

Lung tissue sections were immunostained with a monoclonal antibody against proliferating cell nuclear antigen (PCNA) to assess cell proliferation. The number of cells with nuclei showing immunoreactivity to PCNA was counted, and expressed as the ratio of positive to negative cells in each vessel wall.

\section{Real-time PCR}

Total RNA was extracted from lung tissue using an RNeasy Micro kit (QIAGEN, Barcelona, Spain). Real-time PCR for endothelial nitric oxide synthase (eNOS) and $\beta$-actin was performed as previously described [13]. Results were normalised to $\beta$-actin expression levels and relative gene expression was analysed using the $2^{-\Delta \Delta C t}$ method [20].

\section{Western blot analysis}

eNOS activity was determined in lung homogenates as the ratio between phosphorylated eNOS (PeNOS) and the total eNOS protein expression, analysed by western blot.

\section{Plasma chemistry of vasoactive agents}

A method based on the Griess reaction [21] was used to measure nitrites and nitrates in plasma. ET-1 levels were determined by ELISA. Plasma levels of noradrenaline (NA), adrenaline and serotonin were measured by high-pressure liquid chromatography.

\section{Statistics}

Data in tables are presented as mean \pm SD and data in graphs are presented as mean \pm SEM. A two-way ANOVA was used to evaluate the main effects of $\mathrm{CS}$ and $\mathrm{CH}$, as well as their 
interaction. Post hoc pairwise comparisons were performed using the paired t-test.

\section{RESULTS}

\section{Survival and body weight}

During the first 10 weeks, the mortality rate of animals exposed to CS was $45 \%$, whereas no animal died in the control group. During the final 2 weeks, when hypoxia was added to CS exposure, one animal from each experimental group died, resulting in an additional mortality rate of $6.3 \%$ in the CS, $6.3 \%$ in the $\mathrm{CH}$ and $9.1 \%$ in the $\mathrm{CSCH}$ groups. All the unexposed animals completed the study. The main cause of death $(88 \%$ of cases) was due to bronchoconstriction during CS exposure. Animals that died during the study were excluded from the final analysis.

From week 4, weight gain in the CS group decreased markedly. $\mathrm{CH}$ induced weight loss from the first week of exposure (week 11 of the study). As a result, at the end of the study, body weight in the three experimental groups was lower than in the control group, the $\mathrm{CSCH}$ group showing the lowest value (supplementary fig. 1).

\section{Pulmonary haemodynamics}

CS or hypoxia exposure increased $P$ pa under both normoxic and hypoxic conditions (table 1 and fig. 1a). The increase in $P$ pa in the CS and $\mathrm{CH}$ groups was of similar magnitude, and was much more pronounced in animals subjected to both stimuli (CSCH group) (fig. 1a). Interestingly, Ppa did not change during acute hypoxic challenge in any of the experimental groups, reflecting a lack of hypoxic pulmonary vasoconstriction in this experimental model (fig. 1b). Exposure to hypoxia ( $\mathrm{CH}$ and $\mathrm{CSCH}$ groups) was associated with an increase in SAP (table 1 and fig. 1c).

RV weight increased in animals exposed to hypoxia (table 1) and increased further in animals exposed to hypoxia and CS (CSCH group) (fig. 1d).

At the end of the study, compared with the control group, haematocrit was 7\% higher in the CS group, $5 \%$ in the $\mathrm{CH}$ group and $12 \%$ in the CSCH group (supplementary fig. 2).

\section{Mechanical properties and reactivity of pulmonary artery and aorta}

Vascular distensibility

The distensibility of pulmonary arteries was significantly reduced in animals exposed to hypoxia, the effect being most pronounced in the CSCH group (table 2 and supplementary fig. 3). Overall, the distensibility of the aorta was lower than that of the pulmonary arteries and was similar in all groups.

\section{Contractile responses}

Table 2 shows the vascular responses to potassium chloride $(\mathrm{KCl})$ and NA in the pulmonary artery. Contraction induced by $\mathrm{KCl}$ was greater in pulmonary arteries from animals exposed to $\mathrm{CS}$ or $\mathrm{CH}$, compared with controls ( $\mathrm{p}<0.05$ for both), being further increased when both stimuli were combined (table 2 and supplementary fig. 4). Contraction induced by NA was greater in pulmonary arteries of animals exposed to hypoxia $(\mathrm{CH}$ and $\mathrm{CSCH}$ groups), whereas in the CS group, it was similar to that in the controls (table 2 and supplementary fig. 5). No differences were observed in the contractility of the aorta.

\section{Endothelium-dependent vasorelaxation}

Figure 2 shows the changes in wall tension of the pulmonary artery and aorta induced by cumulative doses of ADP, after pre-contraction with NA. The initial wall tension, after NA precontraction, was higher in pulmonary arteries of animals exposed to hypoxia ( $\mathrm{CH}$ and $\mathrm{CSCH}$ groups). As a result, when evaluating absolute values of wall tension, the area under the relaxation curve (AUC) in these two groups was larger than in the other two groups (control and CS) (table 2). To account for differences in initial wall tension, we evaluated change in tension as percentage of the initial value. After performing this correction, no differences in the AUC of endotheliumdependent responses were observed between groups (table 2). The AUC in the aorta was similar in all groups (fig. 2b).

\section{Pulmonary vascular remodelling}

Wall thickness of pulmonary arteries was greater in the three experimental groups (CS, $\mathrm{CH}$ and $\mathrm{CSCH}$ ) compared with controls. No additive effects were observed when CS and hypoxia were combined (fig. 3a). In contrast, wall thickness in the aorta was not affected by any of the experimental

TABLE 1 Pulmonary haemodynamics and right ventricle hypertrophy

\begin{tabular}{|c|c|c|c|c|c|c|c|}
\hline & \multicolumn{4}{|c|}{ Experimental group } & \multicolumn{3}{|c|}{ Two-way ANOVA main effects $p$-value } \\
\hline & Control & CS & $\mathrm{CH}$ & $\mathrm{CSCH}$ & CS exposure & Hypoxia & Interaction \\
\hline Normoxia & $6.4 \pm 1.7$ & $8.0 \pm 0.7$ & $8.6 \pm 2.6$ & $10.5 \pm 1.8$ & 0.028 & 0.005 & 0.843 \\
\hline Acute hypoxia & $6.2 \pm 2.0$ & $8.2 \pm 1.4$ & $8.2 \pm 2.2$ & $10.2 \pm 1.9$ & 0.016 & 0.016 & 0.994 \\
\hline Fulton index & $0.30 \pm 0.01$ & $0.32 \pm 0.03$ & $0.34 \pm 0.05$ & $0.37 \pm 0.05$ & 0.063 & $<0.001$ & 0.713 \\
\hline
\end{tabular}

Data are presented as mean $\pm \mathrm{SD}$, unless otherwise stated. Normoxia: inspiratory oxygen fraction $\left(\mathrm{Fl}_{1} \mathrm{O}_{2}\right) \quad 0.21$; acute hypoxia: $\mathrm{Fl}, \mathrm{O}_{2} 0.10 ;$ recovery: $\mathrm{Fl}, \mathrm{O}_{2} \quad 0.21$. A p-value $<0.05$ was considered significant. CS: cigarette smoke; $\mathrm{CH}$ : chronic hypoxia; $\mathrm{CSCH}$ : exposure to both $\mathrm{CS}$ and $\mathrm{CH}$; $\bar{P}$ p: mean pulmonary artery pressure; $\mathrm{SAP}$ : systemic arterial pressure. 

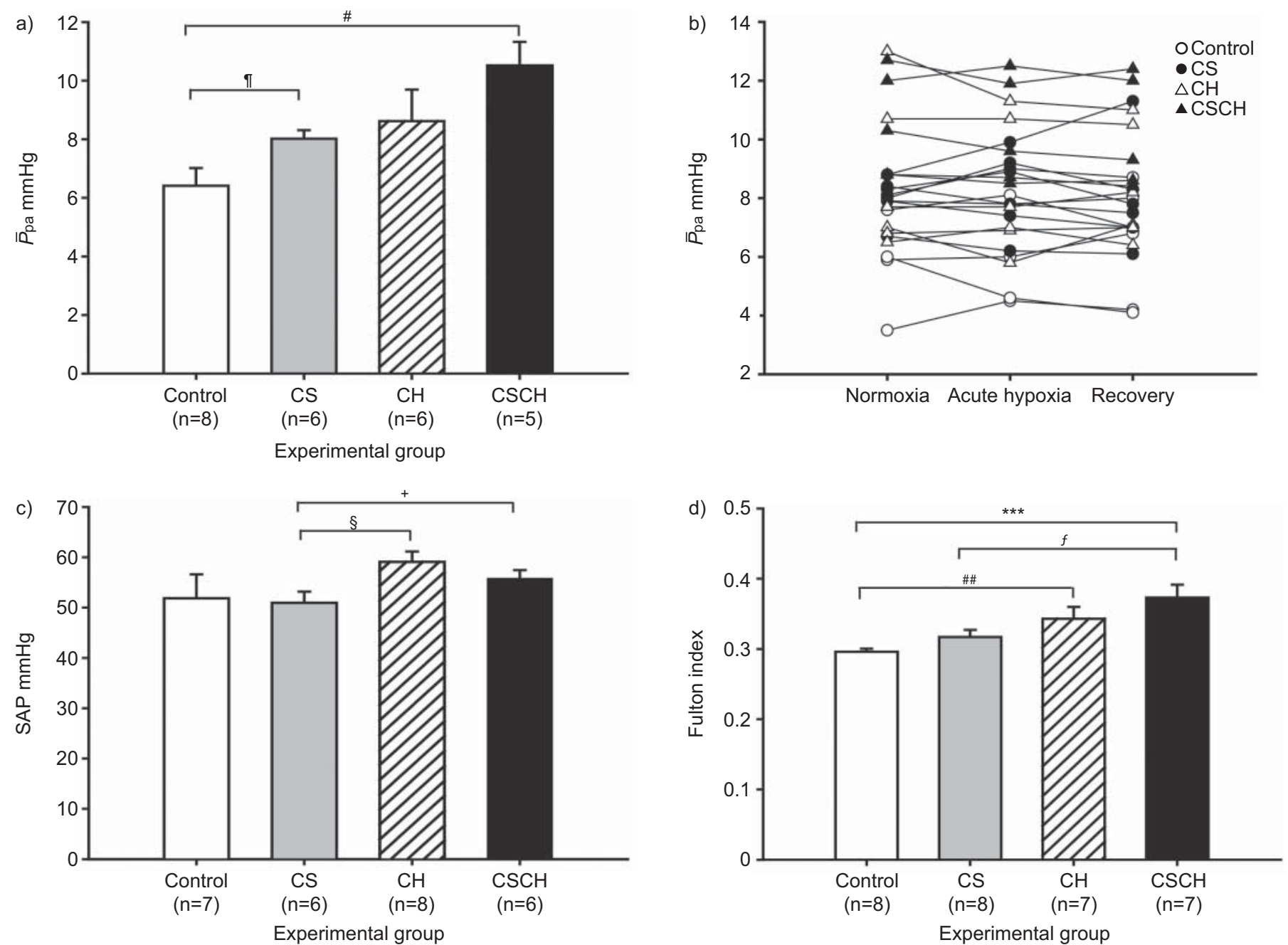

FIGURE 1. Pulmonary and systemic haemodynamics. a) Mean pulmonary artery pressure ( $\bar{p}$ pa) at baseline in control animals, and those exposed to cigarette smoke $(\mathrm{CS})$, chronic hypoxia $(\mathrm{CH})$ or both $(\mathrm{CSCH})$. b) Individual values of $\bar{P}_{\text {pa }}$ in normoxia (inspiratory oxygen fraction $\left(F 1, \mathrm{O}_{2}\right) 0.21$ ), acute hypoxia $\left(F 1, \mathrm{O}_{2} 0.10\right)$ and recovery to normoxia $\left(F \mid \mathrm{O}_{2}\right.$ 0.21). C) Mean systemic arterial pressure (SAP) at baseline. d) Right ventricle (RV) hypertrophy, assessed as the ratio between the RV weight and the left ventricle weight plus the septum weight (Fulton index). Data are presented as mean \pm SEM. ${ }^{\#}: p=0.002 ;{ }^{9}: p=0.05 ;{ }^{+}: p=0.03 ;{ }^{\S}: p=0.007 ;{ }^{f}: p=0.02 ;{ }^{\# \#}: p=0.01$; $* * *: p<0.001$.

conditions (supplementary table 1), although it was thinner in the $\mathrm{CH}$ group compared with the $\mathrm{CS}$ group (fig. $3 \mathrm{~b}$ ).

In animals exposed to hypoxia ( $\mathrm{CH}$ and $\mathrm{CSCH}$ groups) the content of mucopolysaccharides in the main pulmonary artery was increased, whereas collagen was diminished, as compared with the control and CS groups. Elastin content was greater in the CSCH group compared with the control group (fig. 4).

The proportion of small vessels showing positive immunoreactivity to $\mathrm{SM} \alpha$-actin was higher in the three experimental groups, with a highly significant effect for CS exposure (table 3). When arterioles were scored according to the degree of muscularisation, it was apparent that in the $\mathrm{CS}$ and $\mathrm{CSCH}$ groups, there was a decrease in the proportion of nonmuscularised vessels and a concomitant increase in the proportion of fully muscularised intrapulmonary vessels (fig. 5).

Animals exposed to hypoxia ( $\mathrm{CH}$ and $\mathrm{CSCH}$ groups) showed a significantly greater proportion of small intrapulmonary vessels with double elastic laminas compared with control animals (table 3 and supplementary fig. 6).

Assessment of proliferating cells (PCNA-positive/-negative nucleus ratio) in small intrapulmonary vessel walls revealed no differences between groups (supplementary fig. 7). The total number of nuclei per vessel remained unchanged irrespective the type exposure.

\section{Gene expression and activity of eNOS}

The gene expression of eNOS was evaluated by real-time PCR in lung homogenates and normalised to the expression of $\beta$-actin. Lung expression of eNOS was reduced in animals exposed to CS ( $\mathrm{p}=0.028$ for CS effect in ANOVA; supplementary fig. 8a). No additive effect was observed when both stimuli were combined. Changes in PeNOS and eNOS protein expression were evaluated by western blot in lung homogenates. The PeNOS/eNOS ratio was calculated as a measure of the enzyme activity. No significant changes were observed 


\section{TABLE 2 Mechanical properties and reactivity of pulmonary artery}

\begin{tabular}{|c|c|c|c|c|c|c|c|}
\hline & \multicolumn{4}{|c|}{ Experimental group } & \multicolumn{3}{|c|}{ Two-way ANOVA main effects $p$-value } \\
\hline & Control & cs & $\mathrm{CH}$ & $\mathrm{CSCH}$ & CS exposure & Hypoxia & Interaction \\
\hline Subjects $\mathrm{n}$ & 7 & 6 & 7 & 8 & & & \\
\hline Distensibility $\times 10^{-3} \mathrm{mmHg}^{-1}$ & $14.9 \pm 1.9$ & $14.2 \pm 2.9$ & $13.1 \pm 1.7$ & $11.2 \pm 2.8$ & 0.152 & 0.013 & 0.534 \\
\hline \multicolumn{8}{|l|}{ Contraction $\mathrm{mN}$} \\
\hline \multicolumn{8}{|l|}{ Relaxation AUC } \\
\hline \multicolumn{8}{|l|}{ ADP } \\
\hline$\%$ change ${ }^{\#}$ & $207.8 \pm 46.7$ & $171.7 \pm 47.8$ & $176.8 \pm 19.2$ & $205.2 \pm 43.6$ & 0.808 & 0.936 & 0.043 \\
\hline Wall tension & $22.6 \pm 8.5$ & $25.7 \pm 16.2$ & $52.7 \pm 13.2$ & $46.5 \pm 16.2$ & 0.775 & $<0.001$ & 0.406 \\
\hline $\mathrm{ADP}+\mathrm{L}-\mathrm{NAME}^{+}$ & $44.4 \pm 22.5$ & $66.8 \pm 33.9$ & $107.7 \pm 29.7$ & $69.6 \pm 28.3$ & 0.903 & 0.016 & 0.230 \\
\hline $\mathrm{SNP}^{+}$ & $18.4 \pm 8.1$ & $23.6 \pm 12.3$ & $35.4 \pm 8.5$ & $32.1 \pm 13.1$ & 0.820 & 0.005 & 0.316 \\
\hline
\end{tabular}

in the eNOS activity in any of the experimental groups, although a tendency towards a decrease was present in the CS and $\mathrm{CSCH}$ groups (supplementary fig. 8b).

\section{Plasma chemistry of nitrites and nitrates, ET-1, and catecholamines}

Nitrites and nitrates

Plasma levels of nitrites and nitrates decreased in animals exposed to CS, and tended to increase in those exposed to hypoxia (table 4 ).

\section{$E T-1$}

There was strong interaction between the effects of CS and hypoxia on plasma levels of ET-1. Whereas in normoxic animals, ET-1 increased in those exposed to CS, CS reduced ET-1 levels in animals subjected to $\mathrm{CH}$ (table 4).

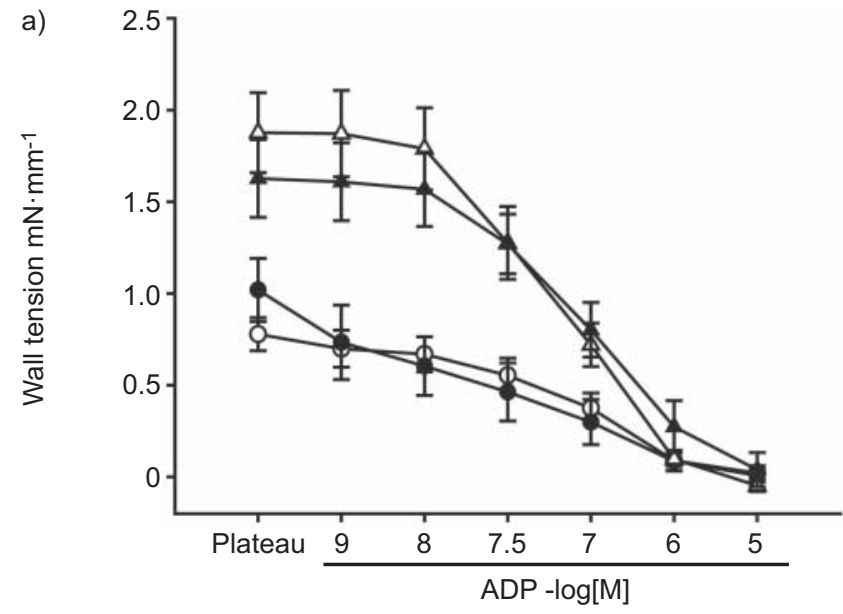

\section{Catecholamines}

There were no significant differences between groups in the plasma levels of NA, adrenaline or serotonin (table 4).

\section{Correlations}

In pulmonary arteries, the contraction in response to $\mathrm{KCl}$ correlated with the vessel wall thickness $(r=0.45 ; \mathrm{p}=0.02)$.

$P$ pa was related to the percentage of SM $\alpha$-actin-positive intrapulmonary vessels. As shown in figure $6 \mathrm{a}, P$ pa increased to similar extent in animals exposed to either $\mathrm{CS}$ or $\mathrm{CH}$, which showed a similar degree of muscularisation, and increased further in those subjected to both exposures ( $\mathrm{CSCH}$ group), which also showed the greatest number of muscularised vessels.

The number of small intrapulmonary vessels positive for SM $\alpha$ actin was inversely correlated with eNOS mRNA expression in

b)

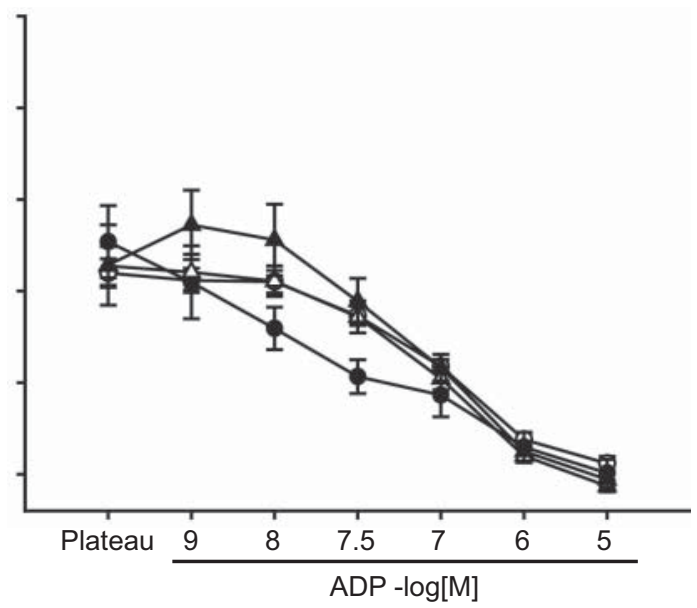

FIGURE 2. Vascular reactivity of a) main pulmonary artery and b) aorta. a) Changes in wall tension of pre-contracted pulmonary artery rings, expressed in millinewtons per millimetre of circumference, in response to cumulative doses of adenosine diphosphate (ADP). b) Changes in wall tension of pre-contracted aorta rings, expressed in millinewtons per millimetre of circumference, in response to cumulative doses of ADP. Data are presented as mean \pm SEM. 

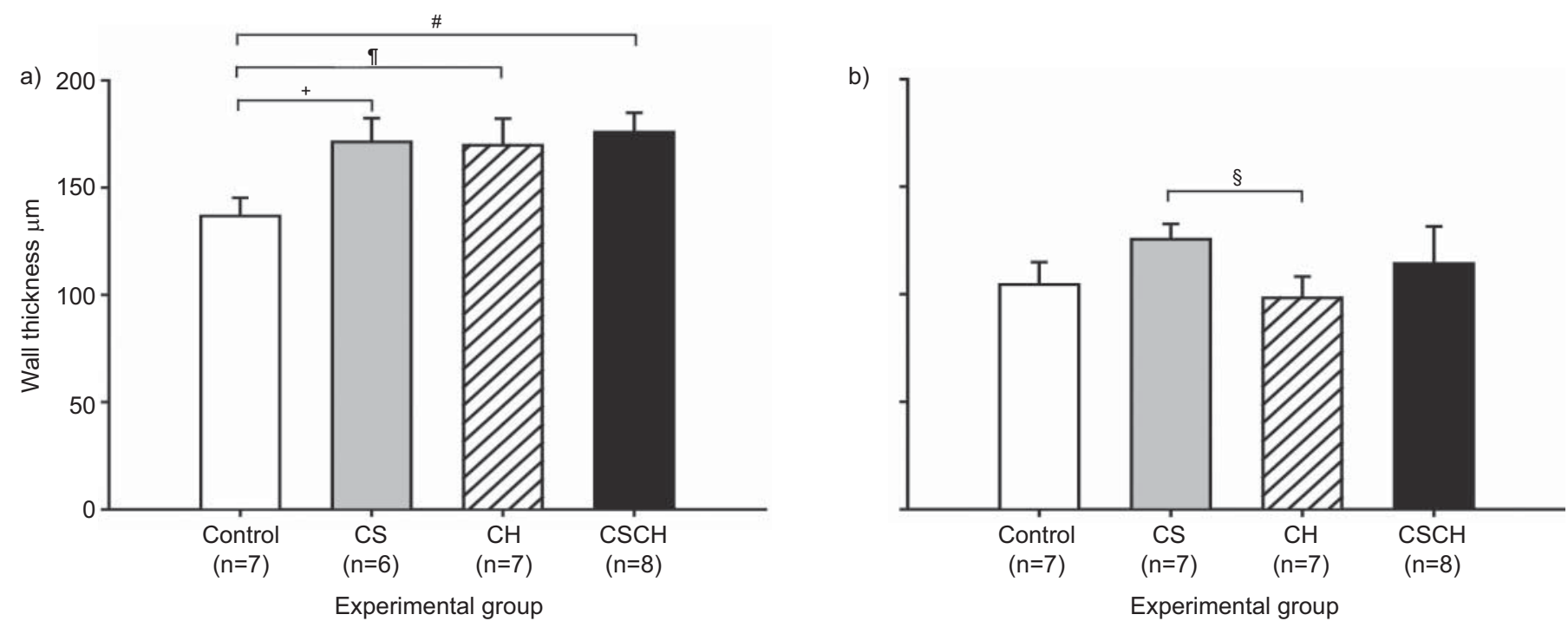

FIGURE 3. Morphometry of a) main pulmonary artery and b) aorta of control animals ( $n=7)$, and those exposed to cigarette smoke (CS; $n=6)$, chronic hypoxia (CH; $n=7)$ or both $(\mathrm{CSCH} ; n=8)$. Data are presented as mean \pm SEM. ${ }^{*}: p=0.008 ;{ }^{\circ}: p=0.005 ;{ }^{+}: p=0.03 ;{ }^{s}: p=0.05$.

lung homogenates (fig. 6b). Moreover, distensibility correlated positively with the mucopolysaccharide content $(\mathrm{r}=0.470$; $\mathrm{p}=0.013)$ and inversely with collagen content $(\mathrm{r}=-0.469$; $\mathrm{p}=0.013)$.

\section{DISCUSSION}

Results of the present study show that both $\mathrm{CS}$ and $\mathrm{CH}$ produced a similar increase in $P$ pa and RV hypertrophy, and that the combination of both agents had a synergistic effect on these alterations. Furthermore, $\mathrm{CS}$ and $\mathrm{CH}$ exerted different effects on the reactivity and mechanical properties of large pulmonary arteries, and on the morphological characteristics of small intrapulmonary vessels.

We have previously hypothesised that the pulmonary vascular changes in COPD start at early disease stages, since they are apparent in patients with moderate disease severity and smokers with normal lung function [2, 3]. Nevertheless, we acknowledge the pivotal role that hypoxia plays in the development of pulmonary hypertension in COPD [22]. Given that COPD patients develop hypoxaemia when airflow obstruction becomes severe, we hypothesised that the effects of hypoxaemia on pulmonary vessels may add to the preexisting effects of CS. In an attempt to mimic this sequence, in the present study, we exposed guinea pigs to a hypoxic environment 10 weeks after initiating CS exposure. In addition, we evaluated the individual effects of CS and hypoxia on pulmonary and systemic vessels, as they have never been compared in the same animal model.

Interestingly, CS and hypoxia exerted similar effects on Ppa, RV hypertrophy and pulmonary vessel remodelling, the effects on $P$ pa and RV hypertrophy being further enhanced when both types of exposure were combined. On average, compared with the values observed in the control group, CS exposure induced a $25 \%$ increase in $P$ pa, exposure to hypoxia a $34 \%$ increase and exposure to both factors a $64 \%$ increase. It is notable that the effect of 12 weeks of CS on Ppa was of a similar magnitude to that induced by 2 weeks of hypoxia, emphasising the prominent effect of CS exposure on pulmonary vessels, which is in agreement with a previous observation made by YAMATO et al. [10]. Yet the current investigation demonstrates that the haemodynamic effects of CS exposure are of a similar magnitude to those produced by hypoxia and that the effects of both factors are further enhanced when they are combined.

The increase in $P$ pa induced by CS or hypoxic exposures might be due to the effects of these agents on pulmonary vessel remodelling, distensibility or tone. Exposure to CS and/or to hypoxia resulted in an increased proportion of muscularised intrapulmonary vessels. Interestingly, the morphological characteristics of muscularised arterioles were nonuniform and appeared to be related to the type of stimulus. Whereas CS exposure was strongly associated with an increased proportion of small vessels showing positive immunostaining for SM $\alpha$ actin, exposure to hypoxia was associated with a greater proportion showing double elastic laminas. These changes were not due to cell proliferation, since the number of cells present in the vessel wall and the proportion of those showing positive immunoreactivity for PCNA did not differ between groups. Our findings are in agreement with those reported by KING et al. [23], who showed ultrastructural differences in SMCs in vessels of rats treated with monocrotaline and rats submitted to hypoxia. While arterioles of monocrotalinetreated rats contained immature SMCs with coarse peripheral myofilaments bounded by thin indistinct elastic laminas, arteriolar SMCs of hypoxic rats were mature, with fine myofilaments and bounded by electron-dense laminas. Therefore, it can be hypothesised that hypoxia may have a stimulating effect on the production of elastin by mature SMCs, whereas CS may induce changes in cell phenotype with increased cytoplasmic content of SM $\alpha$-actin.

Exposure to hypoxia had greater effect on vessel distensibility than CS (table 2), although guinea pigs subjected to both exposures showed the lowest distensibility (table 2 and 


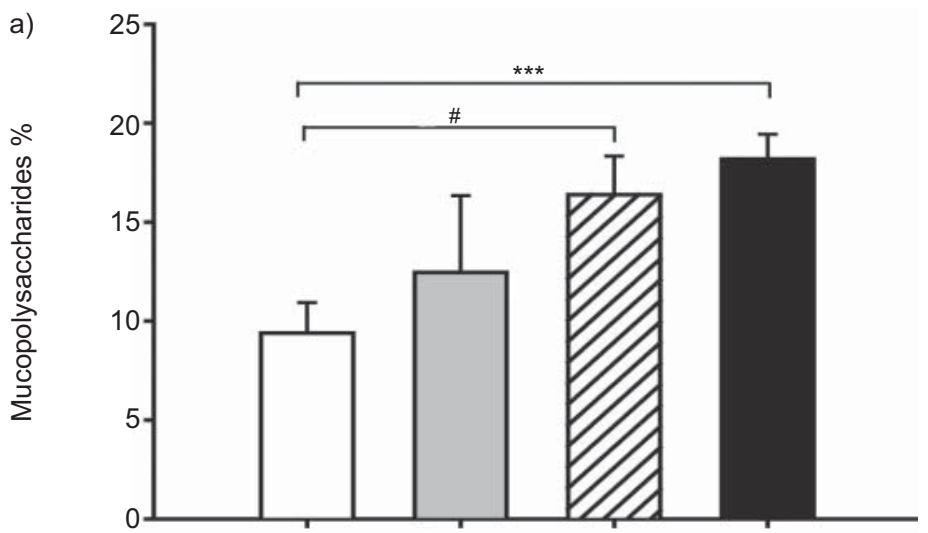

b)
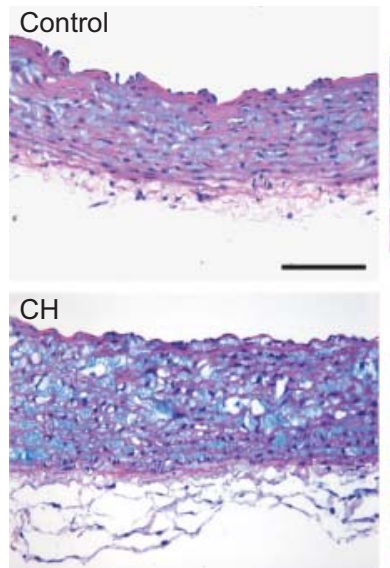

d)

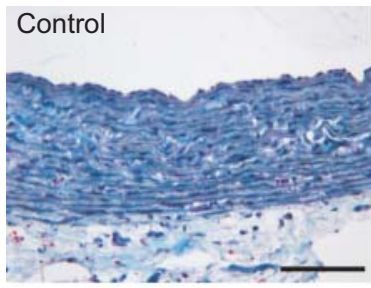

$\mathrm{CH}$

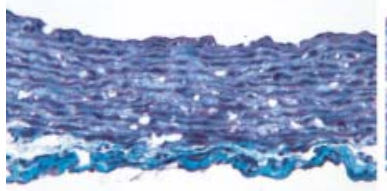

CS

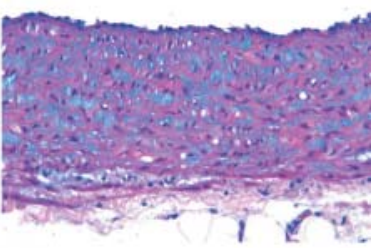

$\mathrm{CSCH}$

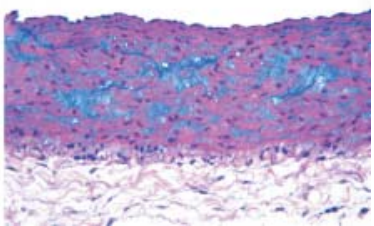

CS

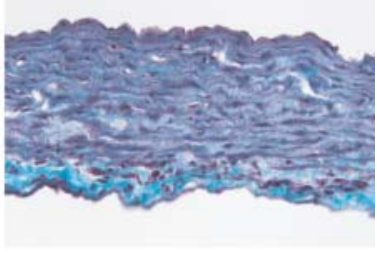

$\mathrm{CSCH}$

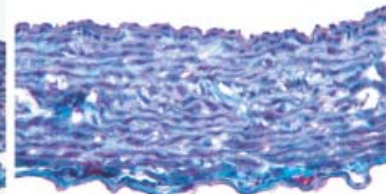

e)

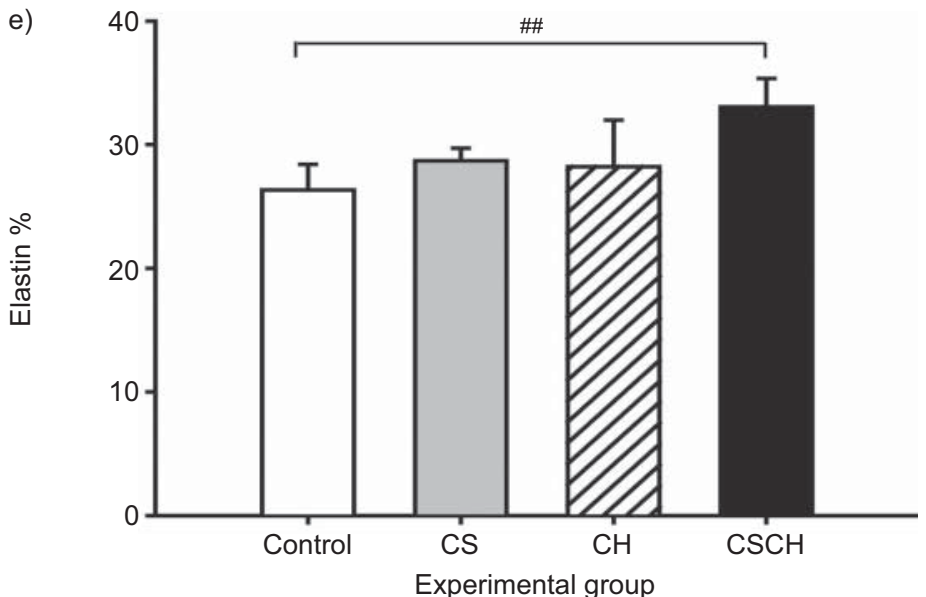

f) Control
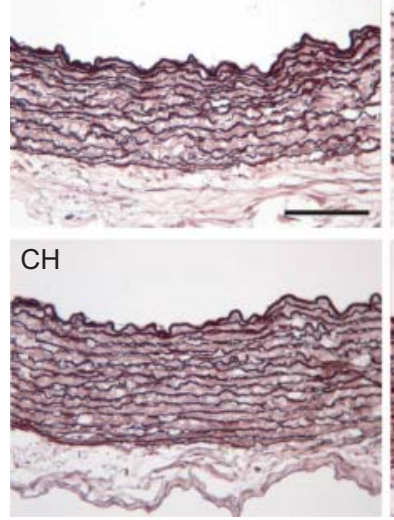

CS

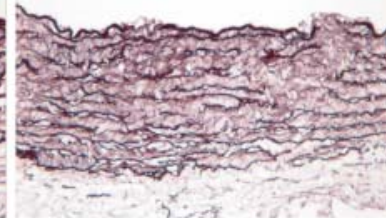

$\mathrm{CSCH}$

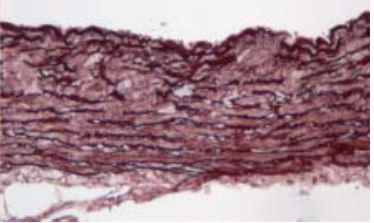

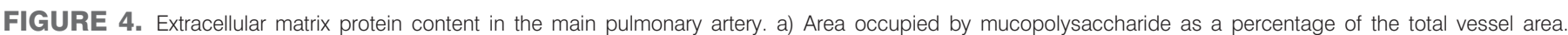

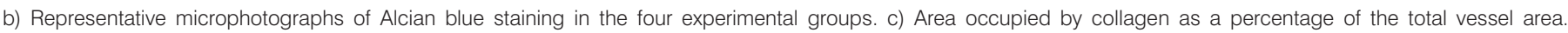

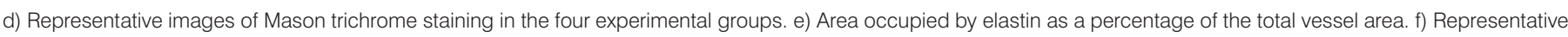
images of orcein staining in the four experimental groups. Data are presented as mean $\pm \mathrm{SEM}$. Scale bars $=50 \mu \mathrm{m}$. CS: cigarette smoke; $\mathrm{CH}$ : chronic hypoxia; $\mathrm{CSCH}$ : exposure to both $\mathrm{CS}$ and $\mathrm{CH}$. ${ }^{* * *}: \mathrm{p}<0.001 ;{ }^{*}: \mathrm{p}=0.016 ;{ }^{\natural}: \mathrm{p}=0.011 ;{ }^{+}: \mathrm{p}=0.012 ;{ }^{\S}: \mathrm{p}=0.035 ;{ }^{f}: \mathrm{p} 0.03 ;{ }^{\# \#}: \mathrm{p}=0.05$.

supplementary fig. 3). The cellular and molecular mechanisms responsible for pulmonary artery stiffening as a result of hypoxia are not well understood. Deposition of extracellular matrix in pulmonary arteries has been shown in animal models of hypoxia-induced pulmonary hypertension [24, 25].
Interestingly, we observed a significant increase in the content of mucopolysaccharides in the wall of main pulmonary arteries, with a concomitant decrease of collagen in the $\mathrm{CH}$ and $\mathrm{CSCH}$ groups, compared with the control group. The content of both proteins correlated with the distensibility of 


\begin{tabular}{|c|c|c|c|c|c|c|c|}
\hline & \multicolumn{4}{|c|}{ Experimental group } & \multicolumn{3}{|c|}{ Two-way ANOVA main effects $p$-value } \\
\hline Subjects $n$ & 8 & 8 & 15 & 13 & & & \\
\hline \multicolumn{8}{|l|}{ Wall thickness $\mu \mathrm{m}$} \\
\hline Pulmonary artery & $137 \pm 22$ & $171 \pm 2$ & $170 \pm 33$ & $176 \pm 26$ & 0.062 & 0.084 & 0.183 \\
\hline \multicolumn{8}{|l|}{ Small intrapulmonary vessels \% } \\
\hline SM $\alpha$-actin-positive arteries & $48.9 \pm 13.0$ & $63.6 \pm 11.0$ & $54.8 \pm 12.5$ & $63.1 \pm 8.7$ & 0.004 & 0.469 & 0.392 \\
\hline Arteries with double elastic lamina & $6.8 \pm 5.1$ & $9.6 \pm 11.8$ & $17.4 \pm 14.5$ & $14.3 \pm 8.6$ & 0.883 & 0.039 & 0.472 \\
\hline
\end{tabular}

Data are presented as mean $\pm \mathrm{SD}$, unless otherwise stated. p-values $<0.05$ were considered significant. $\mathrm{CS}$ : cigarette smoke; $\mathrm{CH}$ : chronic hypoxia; $\mathrm{CSCH}$ : exposure to both $\mathrm{CS}$ and $\mathrm{CH}$; SM: smooth muscle.

pulmonary arteries, suggesting that an imbalance in extracellular matrix protein content may modulate the mechanical properties of the vessels wall.

The increase in Ppa in the experimental groups could also be explained, at least in part, by an increase in vessel tone. In humans, exposure to hypoxia induces pulmonary vasoconstriction. However, in the present study, we did not observe any change in $P$ pa when animals were exposed to acute hypoxia, which is consistent with the lack of hypoxic pulmonary vasoconstriction in the guinea pig [26]. Accordingly, the increased $P_{p a}$ in the $\mathrm{CH}$ group might be explained by

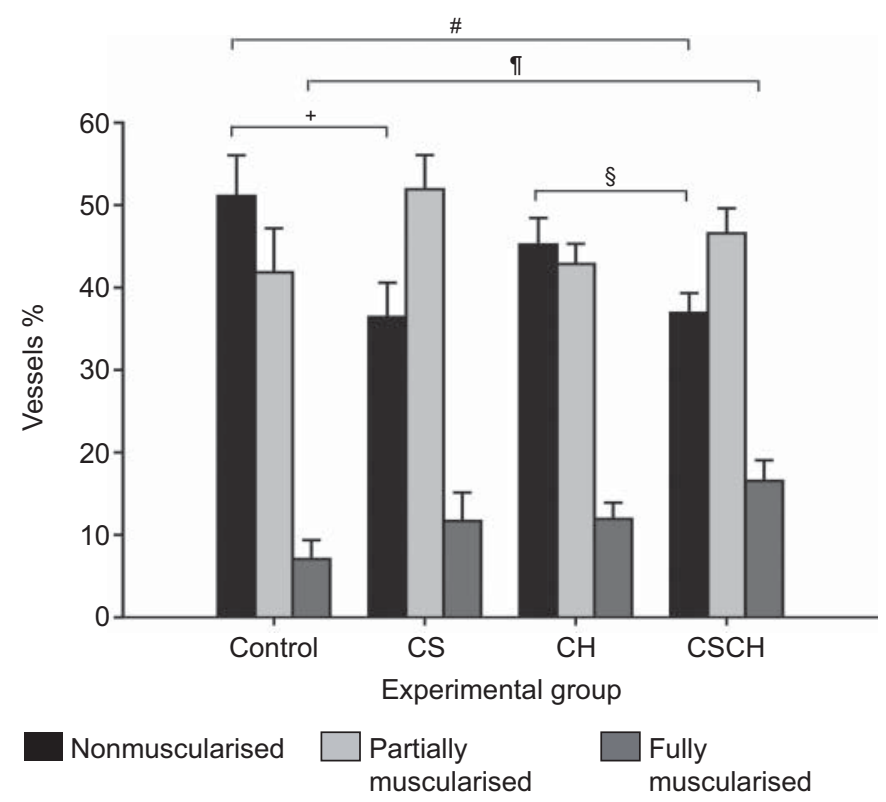

FIGURE 5. Classification of the intrapulmonary arteries according to their degree of muscularisation. Bar charts show the percentage of non-, partially and fully muscularised vessels evaluated in lung sections by immunohistochemistry against smooth muscle $\alpha$-actin. Animals exposed to both cigarette smoke (CS) and chronic hypoxia $(\mathrm{CH})$ ( $\mathrm{CSCH}$ group) developed a greater number of fully muscularised arteries. Data are presented as mean \pm SEM. ${ }^{*}: p=0.009$; ๑: $p=0.02 ;^{+}: p=0.04 ;^{\text {s. }} p=0.05$. adjustments in the contractile/synthetic phenotype of SMC in response to reduced oxygen concentrations [27, 28]. In this respect, it should be noted that in vitro, pulmonary arteries from animals exposed to hypoxia showed greater contraction in response to NA than controls (table 2), which probably reflects changes in SMC adrenergic receptors that regulate vessel tone. Regretfully, we were unable to show a clear increase in the plasma levels of NA and adrenaline in animals exposed to hypoxia (table 4), presumably due to the variability in these measurements and the reduced number of animals in each group. Overall, these results suggest that the effects of hypoxia on the pulmonary circulation exceed those produced by the stimulation of a sympathetic response mediated by peripheral chemoreceptors and baroreceptors in the systemic circulation.

The assessment of endothelium-dependent vasodilatation of pulmonary arteries was largely influenced by the pre-contraction induced by NA, since in arteries from animals exposed to $\mathrm{CH}$, the contraction in response to NA was double that shown in arteries of the control or CS-exposed groups (fig. 2). When ADP-induced relaxation was evaluated as the AUC of the absolute change in tension, values in animals exposed to hypoxia were greater than in the other two groups. However, we do not interpret such a difference as being demonstrative of the impairment of endothelium-dependent relaxation, but as the result of different baseline tension after NA pre-contraction. To account for this difference, we evaluated the change in tension as a percentage of the baseline value. In this case, we did not observe significant differences in the AUC of the relaxation curve among the groups (table 2). Accordingly, we conclude that in the current investigation, neither CS nor exposure to hypoxia produced endothelial dysfunction in pulmonary arteries. In a previous study, we showed that exposure to CS for 3 and 6 months induced endothelial dysfunction in the guinea pig [13]. Differences between the two studies can be explained by the fact that CS exposure in the present study $\left(4\right.$ cigarettes $\cdot$ day $^{-1}$ ) was lower than that used in the previous study ( 7 cigarettes $\cdot$ day $\left.^{-1}\right)$. We used a lower dose of CS because we anticipated greater mortality in animals exposed to a hypoxic environment for 2 weeks after being exposed to CS for 10 weeks, as indeed occurred. The lack of 


\section{TABLE 4 Blood chemistry}

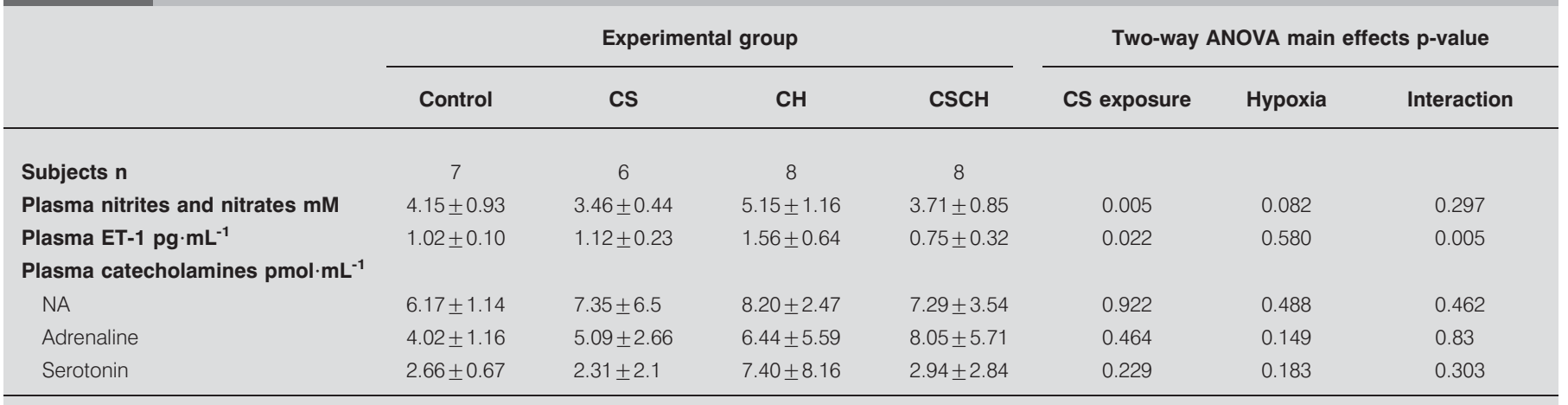

Data are presented as mean $\pm \mathrm{SD}$, unless otherwise stated. p-values $<0.05$ were considered significant. CS: cigarette smoke; $\mathrm{CH}$ : chronic hypoxia; $\mathrm{CSCH}$ : exposure to both CS and $\mathrm{CH}$; ET: endothelin; NA: noradrenaline.
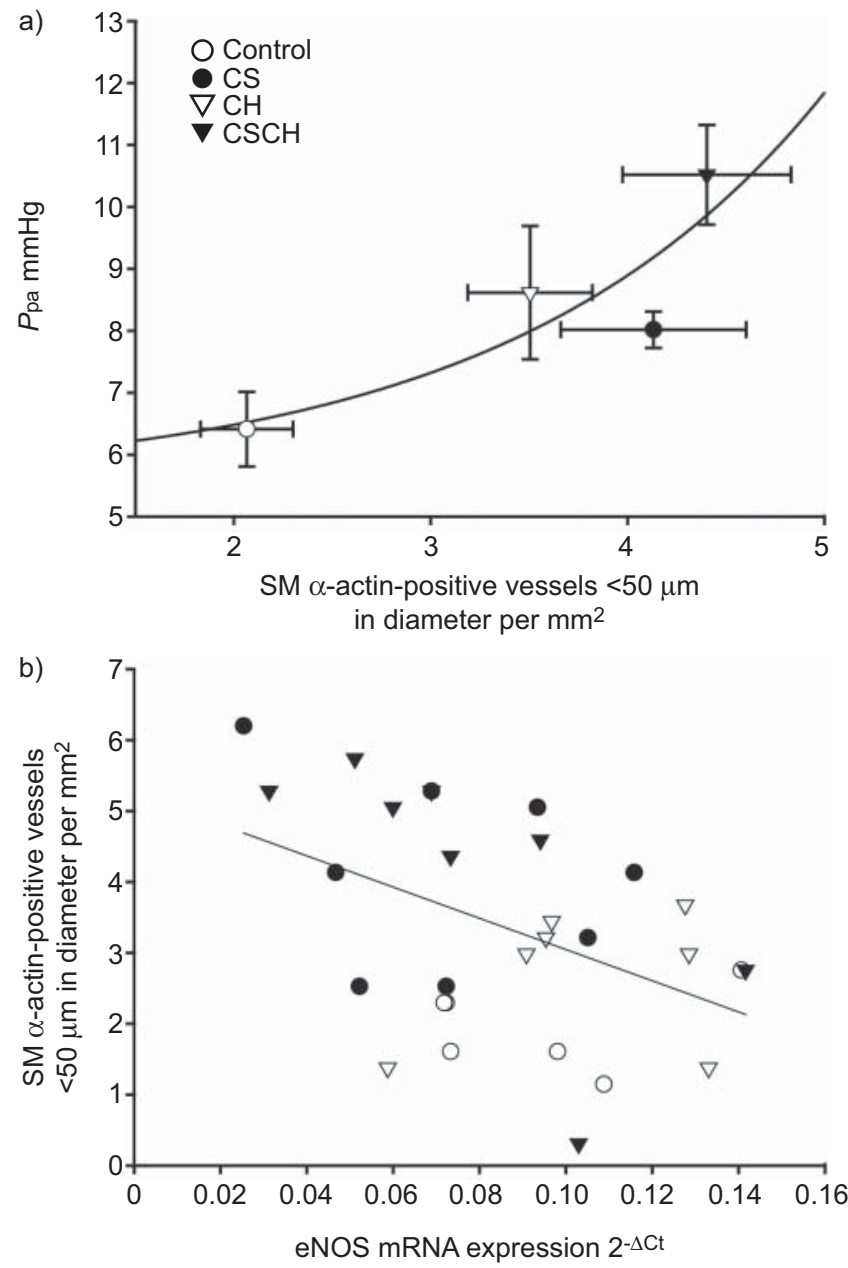

FIGURE 6. a) Plot of pulmonary artery pressure (Ppa) against the degree of vascular remodelling expressed as the number of smooth muscle (SM) $\alpha$-actinpositive vessels $<50 \mu \mathrm{m}$ in diameter per square millimetre of area. The equation was fitted to a modified simple exponential curve. Data are presented as mean \pm SEM. b) Relationship between the MRNA expression of endothelial nitric oxide synthase (eNOS) in lung homogenates and the degree of muscularisation of small intrapulmonary arteries. Lower expression of eNOS is related to a higher content of SM $\alpha$-actin in arteries. $r=0.448 ; p=0.015$. impairment of endothelial function in the main pulmonary arteries contrasts with the observation of decreased expression of eNOS mRNA and protein activity in lung tissue, and reduced plasma levels of nitrites/nitrates in the CS group. We speculate that this situation may represent an initial step that antecedes endothelial dysfunction as assessed in vitro in organ bath chambers. Interestingly, the lung expression of eNOS was inversely related to the SM $\alpha$-actin content in small intrapulmonary vessels, suggesting that vascular cells of these vessels may be a first target of CS, whereas large vessels may require more intense exposure to CS.

We evaluated ET-1 and serotonin levels in plasma because of its actions on SMC physiology and vascular tone. Levels of ET-1 were increased in CS and $\mathrm{CH}$ groups, consistent with the increased levels observed in smokers and COPD patients [29, 30]. However, no differences in ET-1 levels were observed in $\mathrm{CSCH}$ animals, suggesting that the synergistic effect on $P$ pa does not appear to be mediated by ET-1 in plasma.

The greatest effects on $P$ pa and RV hypertrophy were observed in guinea pigs subjected to combined $\mathrm{CS}$ and $\mathrm{CH}$ exposure. Since animals subjected to both factors showed similar vessel remodelling and contractile response to NA, we hypothesise that the greater haemodynamic effect observed in this group can be explained by the combined effects of CS on vessel remodelling and the greater reactivity induced by $\mathrm{CH}$. This observation in the guinea pig is consistent with observations made in COPD, in which patients with moderate disease severity show a similar degree of pulmonary vessel remodelling as patients with severe disease, whereas they differ markedly in arterial oxygen tension $\left(\mathrm{Pa}_{1} \mathrm{O}_{2}\right)$ and $P$ pa [31]. Furthermore, in patients with COPD and chronic respiratory failure, the degree of pulmonary vessel remodelling is not related to the presence of pulmonary hypertension or its severity [32]. Accordingly, our findings suggest that the presence of pulmonary hypertension in COPD, which is commonly associated with chronic respiratory failure, may be due to factors related to hypoxia that add to an underlying process of vessel remodelling produced by cigarette smoking. The different intensity of vessel remodelling and reactivity might explain the great variability in the relationship between $P$ pa and $\mathrm{Pa}_{1} \mathrm{O}_{2}$ observed in COPD [33]. 
Animals exposed to $\mathrm{CS}$ and $\mathrm{CH}$ showed greater haematocrit values, probably due to the additive effects of hypoxia and carboxyhaemoglobin on red cell production. Eventually, the increase in blood viscosity resulting from increased red cell concentration could contribute, to some degree, to the increase in both $P$ pa and SAP. The CSCH group was also the group with least weight gain. Presumably, the mechanisms responsible for lower weight gain could be related to the systemic effects induced either by $\mathrm{CS}$ or $\mathrm{CH}$, akin to those observed in COPD. Body weight was unrelated to mortality, probably because the principal cause of death was bronchoconstriction induced by CS.

Our study has limitations. Cardiac output was not measured due to technical difficulties in its assessment in our experimental setting. Accordingly, we cannot disregard that sympathetic changes might underline some of the differences noted in SAP. Furthermore, the use of anaesthesia during the acute hypoxic challenge might have attenuated hypoxic pulmonary vasoconstriction (HPV) [34]. Accordingly, we cannot completely exclude that the guinea pig may exhibit some degree of $\mathrm{HPV}$, as it has been suggested by THOMPSON et al. [35].

In summary, results of the present study show that in the guinea pig, exposure to $\mathrm{CS}$ or $\mathrm{CH}$ had similar effects on pulmonary hypertension and RV hypertrophy, and when the two factors are combined, the haemodynamic effects are magnified. In animals exposed to CS, mechanisms underlying these haemodynamic changes appear to be related to the remodelling of small intrapulmonary vessels. Exposure to hypoxia modifies the mechanical properties of large pulmonary arteries, presumably by altering extracellular matrix deposition in the vessel wall, enhances their sensitivity to adrenergic agonists, and induces small vessel remodelling. Altogether, this indicates that hypoxaemia represents a critical step in the progression of pulmonary vascular impairment that accelerates and, in some aspects, amplifies the initial effects of CS. These findings contribute to unravelling the mechanisms underlying the development of pulmonary hypertension in COPD, and clarifying the variability in the relationship between arterial oxygenation and pulmonary hypertension.

\section{SUPPORT STATEMENT}

The study was funded by grants from FIS (04/1424), the European Union (2005-018725, Pulmotension), BFU2007-61848 (DGICYT), CIBER CB06/06/0050 (ISCIII) and JCyL-GR242.

\section{STATEMENT OF INTEREST}

None declared.

\section{ACKNOWLEDGEMENTS}

We would like to thank M. de los Llanos Bravo (Dept of Biochemistry and Molecular Biology and Physiology, and IBGM, Universidad de Valladolid, Consejo Superior de Investigaciones Científicas, Valladolid, Spain) and B. González (Dept of Pulmonary Medicine, Hospital ClínicIDIBAPS, Universitat de Barcelona, Barcelona, Spain) for their technical assistance.

\section{REFERENCES}

1 Tozzi CA, Christiansen DL, Poiani GJ, et al. Excess collagen in hypertensive pulmonary arteries decreases vascular distensibility. Am J Respir Crit Care Med 1994; 149: 1317-1326.
2 Santos S, Peinado VI, Ramirez J, et al. Characterization of pulmonary vascular remodelling in smokers and patients with mild COPD. Eur Respir J 2002; 19: 632-638.

3 Peinado VI, Barbera JA, Ramirez J, et al. Endothelial dysfunction in pulmonary arteries of patients with mild COPD. Am J Physiol 1998; 274: L908-L913.

4 Santos S, Peinado VI, Ramirez J, et al. Enhanced expression of vascular endothelial growth factor in pulmonary arteries of smokers and patients with moderate chronic obstructive pulmonary disease. Am J Respir Crit Care Med 2003; 167: 1250-1256.

5 Peinado VI, Barbera JA, Abate P, et al. Inflammatory reaction in pulmonary muscular arteries of patients with mild chronic obstructive pulmonary disease. Am J Respir Crit Care Med 1999; 159: $1605-1611$.

6 Rahman I, Adcock IM. Oxidative stress and redox regulation of lung inflammation in COPD. Eur Respir J 2006; 28: 219-242.

7 Le Cras TD, McMurtry IF. Nitric oxide production in the hypoxic lung. Am J Physiol Lung Cell Mol Physiol 2001; 280: L575-L582.

8 Nicolls MR, Voelkel NF. Hypoxia and the lung: beyond hypoxic vasoconstriction. Antioxid Redox Signal 2007; 9: 741-743.

9 Stenmark KR, Davie NJ, Reeves JT, et al. Hypoxia, leukocytes, and the pulmonary circulation. J Appl Physiol 2005; 98: 715-721.

10 Yamato H, Churg A, Wright JL. Guinea pig pulmonary hypertension caused by cigarette smoke cannot be explained by capillary bed destruction. J Appl Physiol 1997; 82: 1644-1653.

11 Wright JL, Tai H, Churg A. Vasoactive mediators and pulmonary hypertension after cigarette smoke exposure in the guinea pig. J Appl Physiol 2006; 100: 672-678.

12 Wright JL, Churg A. Short-term exposure to cigarette smoke induces endothelial dysfunction in small intrapulmonary arteries: analysis using guinea pig precision cut lung slices. J Appl Physiol 2008; 104: 1462-1469.

13 Ferrer E, Peinado VI, Diez M, et al. Effects of cigarette smoke on endothelial function of pulmonary arteries in the guinea pig. Respir Res 2009; 10: 76.

14 Thompson BT, Steigman DM, Spence CL, et al. Chronic hypoxic pulmonary hypertension in the guinea pig: effect of three levels of hypoxia. J Appl Physiol 1993; 74: 916-921.

15 Underwood DC, Bochnowicz S, Osborn RR, et al. Nonpeptide endothelin receptor antagonists. X. Inhibition of endothelin-1- and hypoxia-induced pulmonary pressor responses in the guinea pig by the endothelin receptor antagonist, SB 217242. J Pharmacol Exp Ther 1997; 283: 1130-1137.

16 Ardite E, Peinado VI, Rabinovich RA, et al. Systemic effects of cigarette smoke exposure in the guinea pig. Respir Med 2006; 100: 1186-1194.

17 Fischer EC, Santana DB, Zocalo Y, et al. Effects of removing the adventitia on the mechanical properties of ovine femoral arteries in vivo and in vitro. Circ J 2010; 74: 1014-1022.

18 Brouwers-Ceiler DL, Nelissen-Vrancken HJ, Smits JF, et al. The influence of angiotensin II-induced increase in aortic wall mass on compliance in rats in vivo. Cardiovasc Res 1997; 33: 478-484.

19 Crosby A, Jones FM, Southwood M, et al. Pulmonary vascular remodeling correlates with lung eggs and cytokines in murine schistosomiasis. Am J Respir Crit Care Med 2010; 181: 279-288.

20 Livak KJ, Schmittgen TD. Analysis of relative gene expression data using real-time quantitative PCR and the $2^{-\Delta \Delta C t}$ method. Methods 2001; 25: 402-408.

21 Granger DL, Taintor RR, Boockvar KS, et al. Measurement of nitrate and nitrite in biological samples using nitrate reductase and Griess reaction. Methods Enzymol 1996; 268: 142-151.

22 Weitzenblum E, Hirth C, Ducolone A, et al. Prognostic value of pulmonary artery pressure in chronic obstructive pulmonary disease. Thorax 1981; 36: 752-758.

23 King A, Smith P, Heath D. Ultrastructural differences between pulmonary arteriolar muscularization induced by hypoxia and monocrotaline. Exp Mol Pathol 1994; 61: 24-35. 
24 Kobs RW, Muvarak NE, Eickhoff JC, et al. Linked mechanical and biological aspects of remodeling in mouse pulmonary arteries with hypoxia-induced hypertension. Am J Physiol Heart Circ Physiol 2005; 288: H1209-H1217.

25 Bank AJ, Wang H, Holte JE, et al. Contribution of collagen, elastin, and smooth muscle to in vivo human brachial artery wall stress and elastic modulus. Circulation 1996; 94: 3263-3270.

26 Schwenke DO, Bolter CP, Cragg PA. Are the carotid bodies of the guinea-pig functional? Comp Biochem Physiol A Mol Integr Physiol 2007; 146: 180-188.

27 Rensen SS, Doevendans PA, van Eys GJ. Regulation and characteristics of vascular smooth muscle cell phenotypic diversity. Neth Heart J 2007; 15: 100-108.

28 Owens GK, Kumar MS, Wamhoff BR. Molecular regulation of vascular smooth muscle cell differentiation in development and disease. Physiol Rev 2004; 84: 767-801.

29 Goerre S, Staehli C, Shaw S, et al. Effect of cigarette smoking and nicotine on plasma endothelin-1 levels. J Cardiovasc Pharmacol 1995; 26: Suppl. 3, S236-S238.
30 Spiropoulos K, Trakada G, Nikolaou E, et al. Endothelin-1 levels in the pathophysiology of chronic obstructive pulmonary disease and bronchial asthma. Respir Med 2003; 97: 983-989.

31 Magee F, Wright JL, Wiggs BR, et al. Pulmonary vascular structure and function in chronic obstructive pulmonary disease. Thorax 1988; 43: 183-189.

32 Wright JL, Petty T, Thurlbeck WM. Analysis of the structure of the muscular pulmonary arteries in patients with pulmonary hypertension and COPD: National Institutes of Health nocturnal oxygen therapy trial. Lung 1992; 170: 109-124.

33 Scharf SM, Iqbal M, Keller C, et al. Hemodynamic characterization of patients with severe emphysema. Am J Respir Crit Care Med 2002; 166: 314-322.

34 Busch CJ, Spohr FA, Motsch J, et al. Effects of ketamine on hypoxic pulmonary vasoconstriction in the isolated perfused lungs of endotoxaemic mice. Eur J Anaesthesiol 2010; 27: 61-66.

35 Thompson BT, Hassoun PM, Kradin RL, et al. Acute and chronic hypoxic pulmonary hypertension in guinea pigs. J Appl Physiol 1989; 66: 920-928. 